Supporting Information

\title{
CUT-LAMP: Contamination-Free Loop-Mediated Isothermal Amplification Based on the CRISPR/Cas9 Cleavage
}

Yijuan Bao, ${ }^{\dagger, \#}$ Yongzhong Jiang, ${ }^{\mathbb{P},},{ }^{\ddagger}, \#$ Erhu Xiong, ${ }^{*}{ }^{\dagger}$ Tian Tian, ${ }^{\dagger}$ Zhenzhen Zhang, ${ }^{\S}$ Jing Lv, ${ }^{\ddagger}$ Yang $\mathrm{Li},{ }^{*, \ddagger}$ and Xiaoming Zhou*,†

${ }^{\dagger}$ College of Biophotonics \& School of Life Sciences, South China Normal University, Guangzhou 510631, P. R. China

PKey Laboratory of Analytical Chemistry for Biology and Medicine (Ministry of Education), College of Chemistry and Molecular Sciences, Wuhan University, Wuhan 430072, P. R. China

${ }^{\ddagger}$ Hubei Provincial Center for Disease Control and Prevention, Wuhan 430079, P. R. China

${ }^{\S}$ Institute of Human Virology, Zhongshan School of Medicine, Sun Yat-Sen University, Guangzhou 510275, P. R. China

${ }^{\#}$ Y.J. Bao and Y.Z. Jiang contributed equally to this work. 


\section{Experimental Section}

Cas9 Expression and Purification. The pET28a/Cas9-Cys was a gift from Hyongbum Kim (Addgene plasmid \#53261; http://n2t.net/addgene:53261; RRID: Addgene_53261). Plasmid pET28a/Cas9-Cys was transformed into Escherichia coli Rosetta 2 (DE3). E. coli was shocked (250 rpm/min) for $4 \mathrm{~h}$ in TB-kanamycin $(30 \mu \mathrm{g} / \mathrm{mL})$ medium at $37^{\circ} \mathrm{C}$. Once the OD $_{600}$ reached $0.6-0.8$, IPTG $(1 \mathrm{mM})$ was supplied to induce Cas9 expression at $18{ }^{\circ} \mathrm{C}$ overnight. Bacterial cultures were then harvested and resuspend in lysis buffer (20 mM TrisHCl, pH 7.5, 1 mM NaCl, 10\% glycerin), followed by supersonic treatment on ice for 6 mins. Then, lysate was centrifuged at $4{ }^{\circ} \mathrm{C}$ and supernatants were collected. The protein was purified and eluted through Ni-NTA affinity chromatography. The purity of Cas9 was explained by 6\% SDS-PAGE gel scan analysis.

Synthesis of sgRNAs. DNA template that contains sgRNA-encoding sequence and T7 promoter sequence was prepared by a fill-in PCR reaction. PCR reaction system was consisted of PrimeSTAR DNA Polymerase mixture, a forward customized primer containing the T7 promoter and the targeting sequence, and a reverse universal primer encoding the sgRNA scaffold. The two-step PCR was performed with the following cycling conditions: 33 cycles of $98{ }^{\circ} \mathrm{C}$ for $10 \mathrm{~s}$ and $68{ }^{\circ} \mathrm{C}$ for $10 \mathrm{~s}$. PCR product was verified by gel electrophoresis and purified using SanPrep Column PCR Product Purification Kit. This purified product was transcribed (T7 polymerase, NTPs and RNAase inhibitor) at $37{ }^{\circ} \mathrm{C}$ for $4 \mathrm{~h}$ in transcription buffer (40 mM Tris-HCl, $6 \mathrm{mM} \mathrm{MgCl}_{2}, 1 \mathrm{mM}$ DTT, $2 \mathrm{mM}$ spermidine) to create sgRNA. After transcription, sgRNAs were purified by RNA Purification Kit and quantified by the NanoDrop 2000.

Synthesis of crRNAs. DNA template which contains crRNA-encoding sequence and T7 promoter sequence was synthesized by annealing two complementary oligonucleotides (10 $\mu \mathrm{M})$. DNA template was heated at $95{ }^{\circ} \mathrm{C}$ for $5 \mathrm{~min}$ and then cooled down to $25{ }^{\circ} \mathrm{C}$ with $2{ }^{\circ} \mathrm{C} / \mathrm{min}$ in $1 \times$ hybridization buffer (20 mM Tris-HCl, $\mathrm{pH}$ 7.5, $100 \mathrm{mM} \mathrm{KCl,} 5 \mathrm{mM} \mathrm{MgCl}$ ). 
Annealed product was purified and served as template to produce a crRNA with T7 polymerase, NTPs and RNAase inhibitor at $37^{\circ} \mathrm{C}$ for $4 \mathrm{~h}$ in transcription buffer ( $40 \mathrm{mM}$ Tris$\mathrm{HCl}, 6 \mathrm{mM} \mathrm{MgCl}_{2}$, $1 \mathrm{mM}$ DTT, $2 \mathrm{mM}$ spermidine). Transcribed RNAs were purified by RNA purification kit and subsequently stored at $-80^{\circ} \mathrm{C}$. The concentration of the obtained crRNA was measured and quantified by Nanodrop 2000 .

Bacterial Culture and DNA Extraction. Salmonella enterica and Methicillin-resistant staphylococcus aureus were kept in our Laboratory collection. Cultures were grown in Luria Bertani (LB) medium (10 g/L tryptone, $5 \mathrm{~g} / \mathrm{L}$ yeast extract and $5 \mathrm{~g} / \mathrm{L} \mathrm{NaCl})$ at $37{ }^{\circ} \mathrm{C}$ for approximately 12-18 h. Genomic DNA was extracted by Bacteria Genomic DNA Extraction Kit, and quantitatively determined by $1 \%$ agarose gel electrophoresis. Concentration of genomic DNAs was measured by NanoDrop 2000, and genomic DNAs was then stored at $20^{\circ} \mathrm{C}$. The genomic DNAs from $N$. meningitidis were obtained from Hubei provincial centre for Disease Control and Prevention. The Genomic DNAs of Zika was obtained from Sun YatSen University.

In vitro CRISPR/Cas9 Cleavage and Characterization. Cas9 and sgRNA were preincubated in cleavage buffer (20 mM HEPES, $100 \mathrm{mM} \mathrm{NaCl,} 5 \mathrm{mM} \mathrm{MgCl} 2,0.1 \mathrm{mM}$ EDTA) for 10 min at $25{ }^{\circ} \mathrm{C}$ to formed a binary complex. $1 \mu \mathrm{L}$ of target DNA was then added to Cas9/sgRNA mixture in $10 \mu \mathrm{L}$ system. The mixture was incubated at $25{ }^{\circ} \mathrm{C}$ or $37^{\circ} \mathrm{C}$ for 30 min. The assay was stopped by heating at $65{ }^{\circ} \mathrm{C}$ for $10 \mathrm{~min}$. CRISPR/Cas9 cleavage products were evaluated with fragment analysis by 10\% PAGE at $140 \mathrm{~V}$ for $1 \mathrm{~h}$ followed by silver staining. 


\section{Supporting Figures}

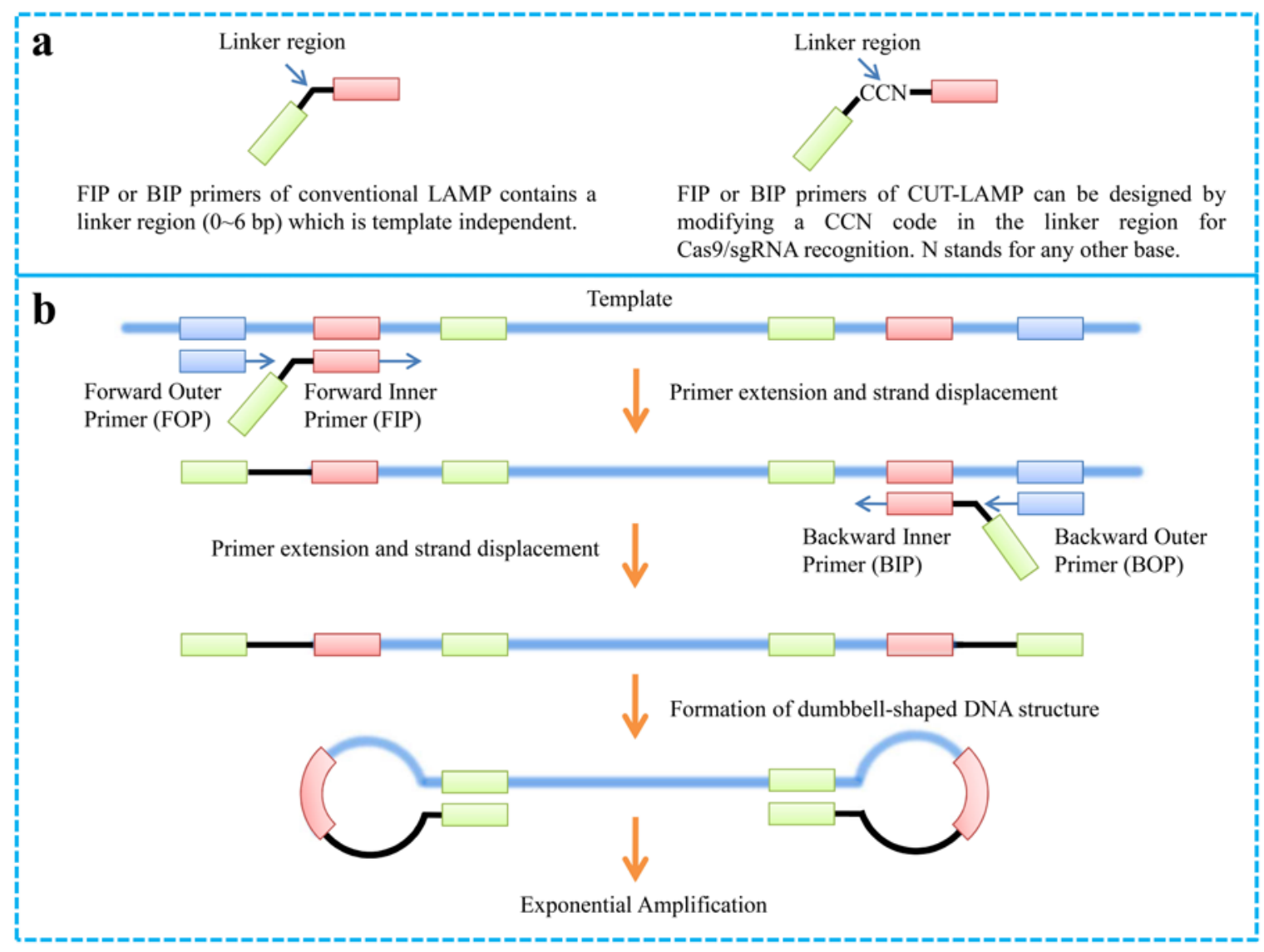

Figure S1. (a) Structure comparison of FIP or BIP of the CON-LAMP and the CUT-LAMP reaction. (b) Process of generating dumbbell-shaped DNA structure in the CUT-LAMP reaction. The dumbbell-shaped DNA structure is used as a template for exponential amplification. Since the dumbbell-shaped DNA structure includes a CCN code, so the LAMP products will contain a PAM site-NGG code near the sgRNA target region. 

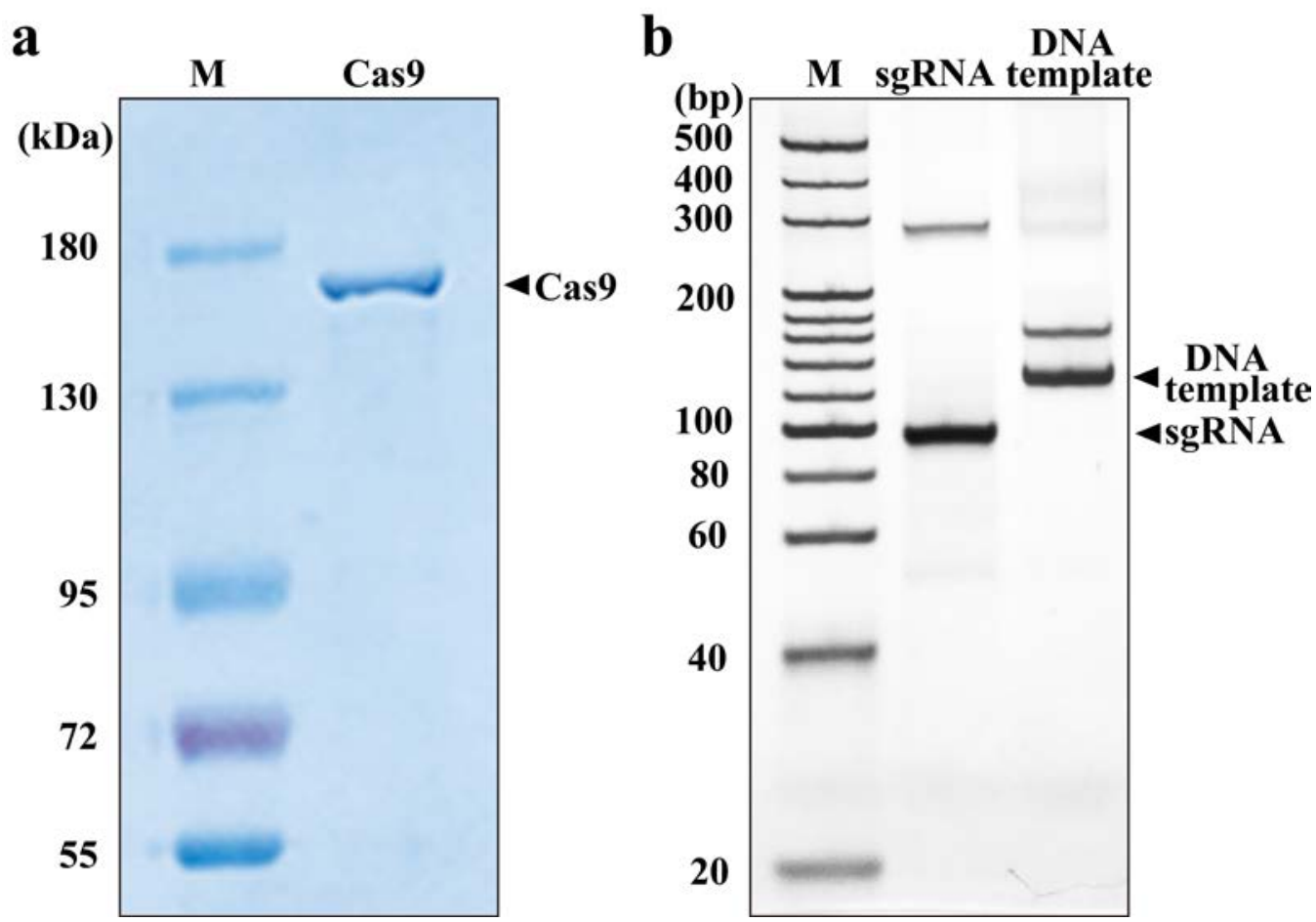

Figure S2. 6\% SDS-PAGE electrophoresis characterization of the home-made Cas9 protein and 10\% native PAGE electrophoresis characterization of sgRNA. Two bands are appeared in sgRNA electrophoresis. They may represent the native single-stranded RNA and a secondary structure formed by sgRNA. 

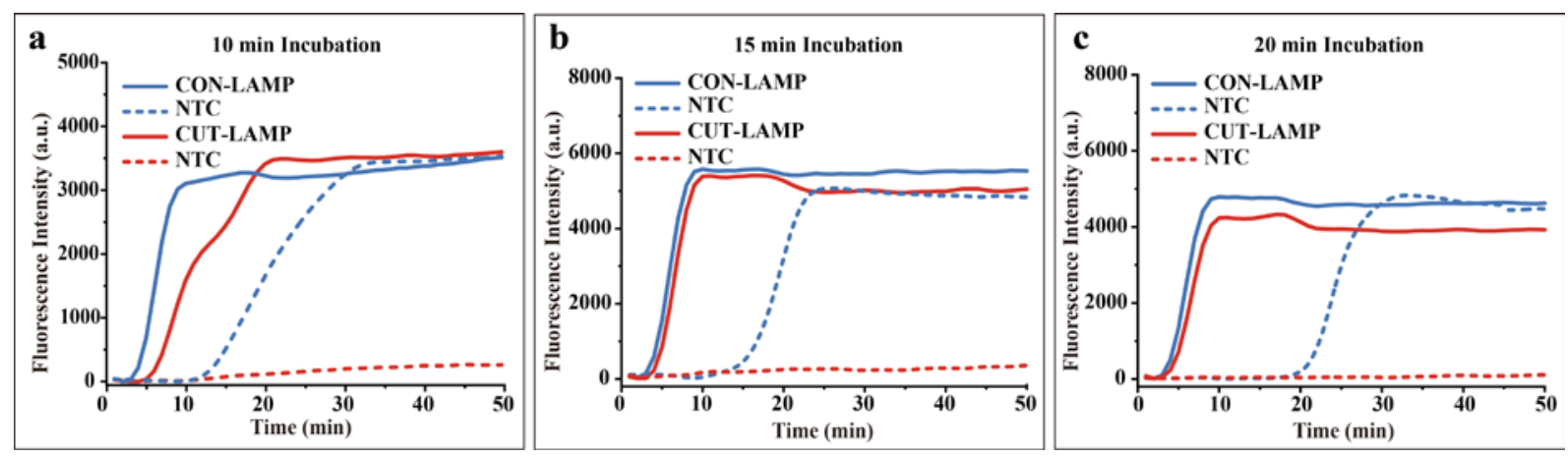

Figure S3. Test of cleavage ability of the CUT-LAMP reaction with various pre-incubation time. Compared to the CON-LAMP, the CUT-LAMP contains a pre-incubation step before amplification. In the presence of $10 \mathrm{pg}$ contaminants, different pre-incubation time of 5 min (Figure 4c), (a) $10 \mathrm{~min}$, (b) $15 \mathrm{~min}$, and (c) 20 min were evaluated, and the results indicate that the false-positive amplification caused by contaminants disappeared with 5 min preincubation. NTC stands for no template control. 


\begin{tabular}{|c|c|}
\hline 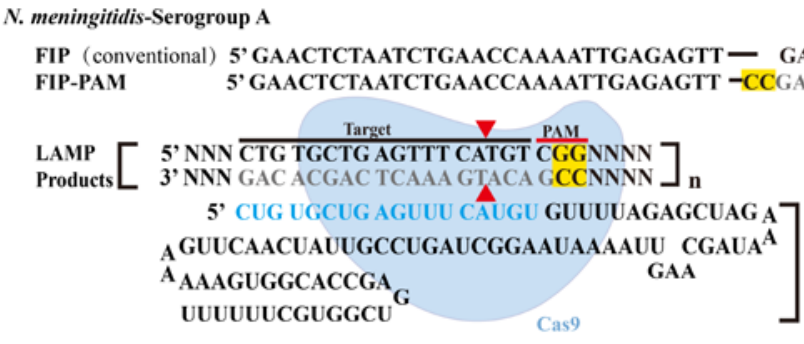 & $\begin{array}{l}\text { 'CATGAAACTCAGCACAG 3, } \\
\text { CATGAAACTCAGCACAG 3' } \\
\text { sgRNA }\end{array}$ \\
\hline 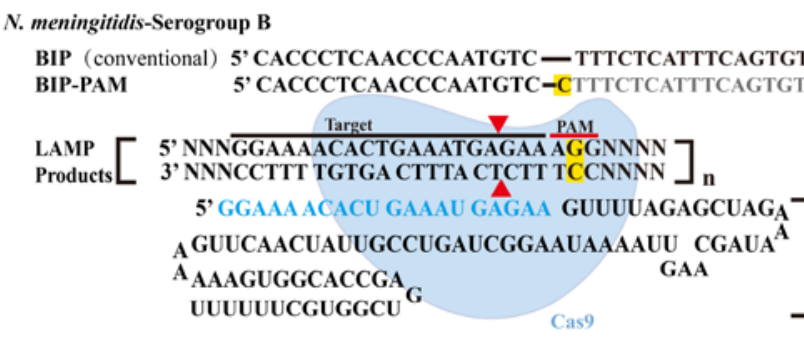 & $\begin{array}{l}\text { ITTTCC 3, } \\
\text { TTTCC 3', } \\
\text { sgRNA }\end{array}$ \\
\hline 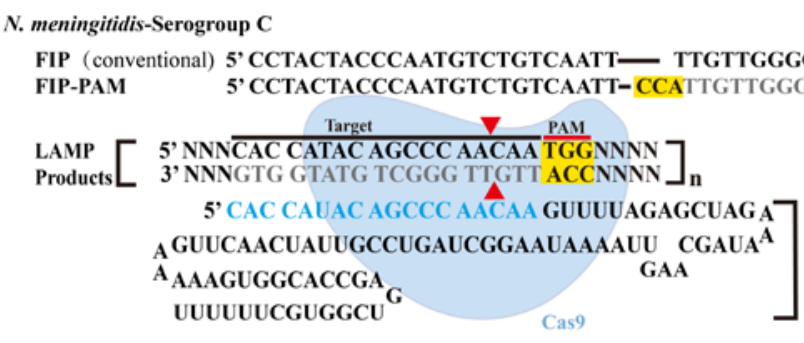 & $\begin{array}{l}\text { CTGTATGGTG 3, } \\
\text { SCTGTATGGTG } 3 \text {, } \\
\text { GQRNA }\end{array}$ \\
\hline 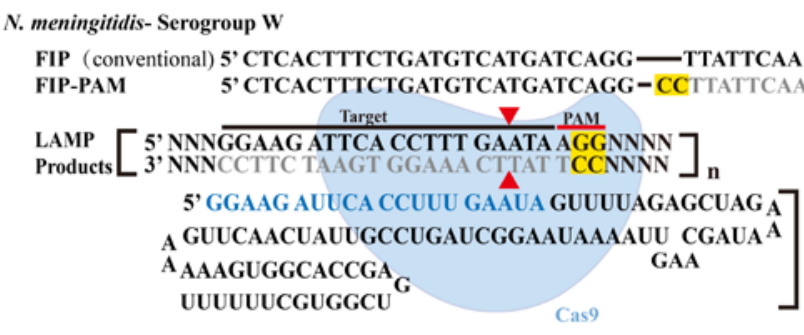 & $\begin{array}{l}\text { AGGTGAATCTTCCGA } 3, \\
\text { AGGTGAATCTTCCGA } 3 \text {, } \\
\text { sgRNA }\end{array}$ \\
\hline 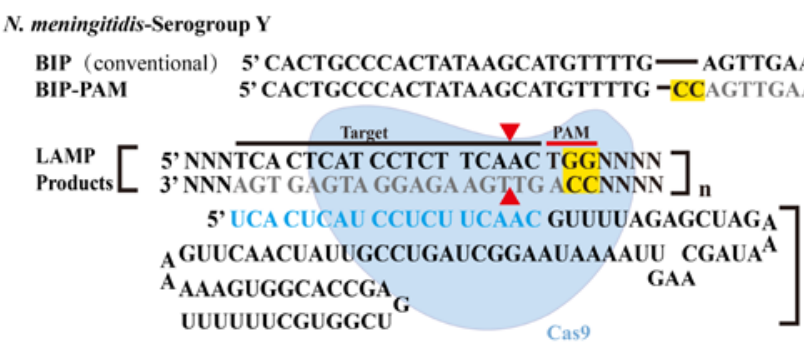 & $\begin{array}{l}\text { AGAGGATGAGTGA 3, } \\
\text { AGAGGATGAGTGA } 3 \text {, } \\
\\
\text { sgRNA }\end{array}$ \\
\hline
\end{tabular}

Figure S4. Sequences of the conventional FIPs or BIPs and PAM site-modified FIPs or BIPs, and the corresponding sgRNA sequences for targeting the sequence adjacent to the PAM site 
to detect five kinds of $N$. meningitidis serotypes by using the CUT-LAMP methods. The PAM sites are highlighted in yellow and the red arrows indicate the cutting position of Cas9/sgRNA. 

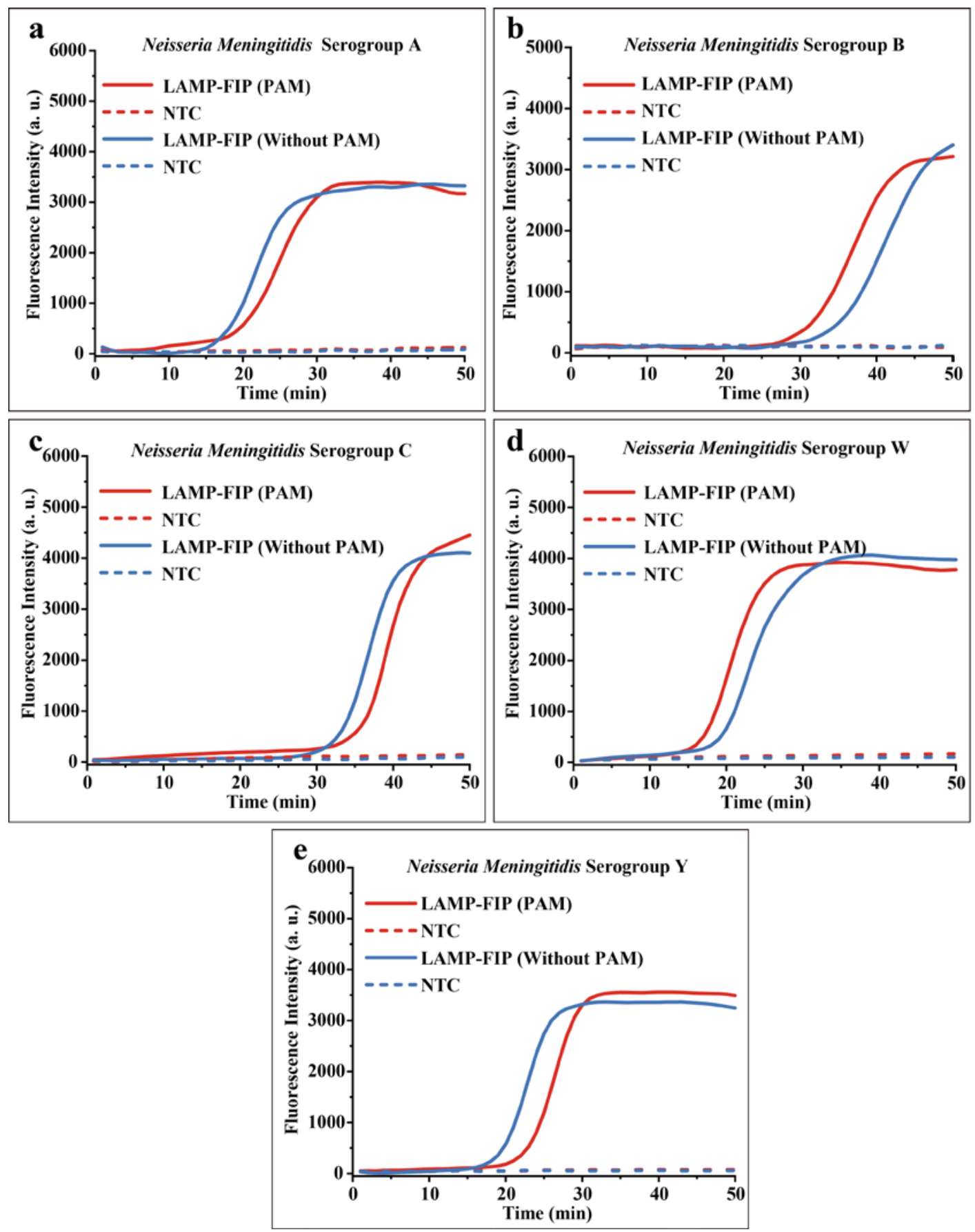

Figure S5. Test of the CON-LAMP amplification efficiency and specificity by using conventional FIPs or BIPs without PAM site and modified FIPs or BIPs with PAM site. (a) The LAMP amplification of $s a c B$ gene of $N$. meningitidis serogroup A by using conventional primer and modified primer. (b) The LAMP amplification of siaD gene of $N$. meningitidis serogroup B by using conventional primer and modified primer. (c) The LAMP amplification of siaD gene of $N$. meningitidis serogroup C by using conventional primer and modified primer. (d) The LAMP amplification of synG gene of $N$. meningitidis serogroup W by using 
conventional primer and modified primer. (e). The LAMP reaction of synF gene of $N$. meningitidis serogroup Y by using conventional primer and modified primer. NTC stands for no template control. 

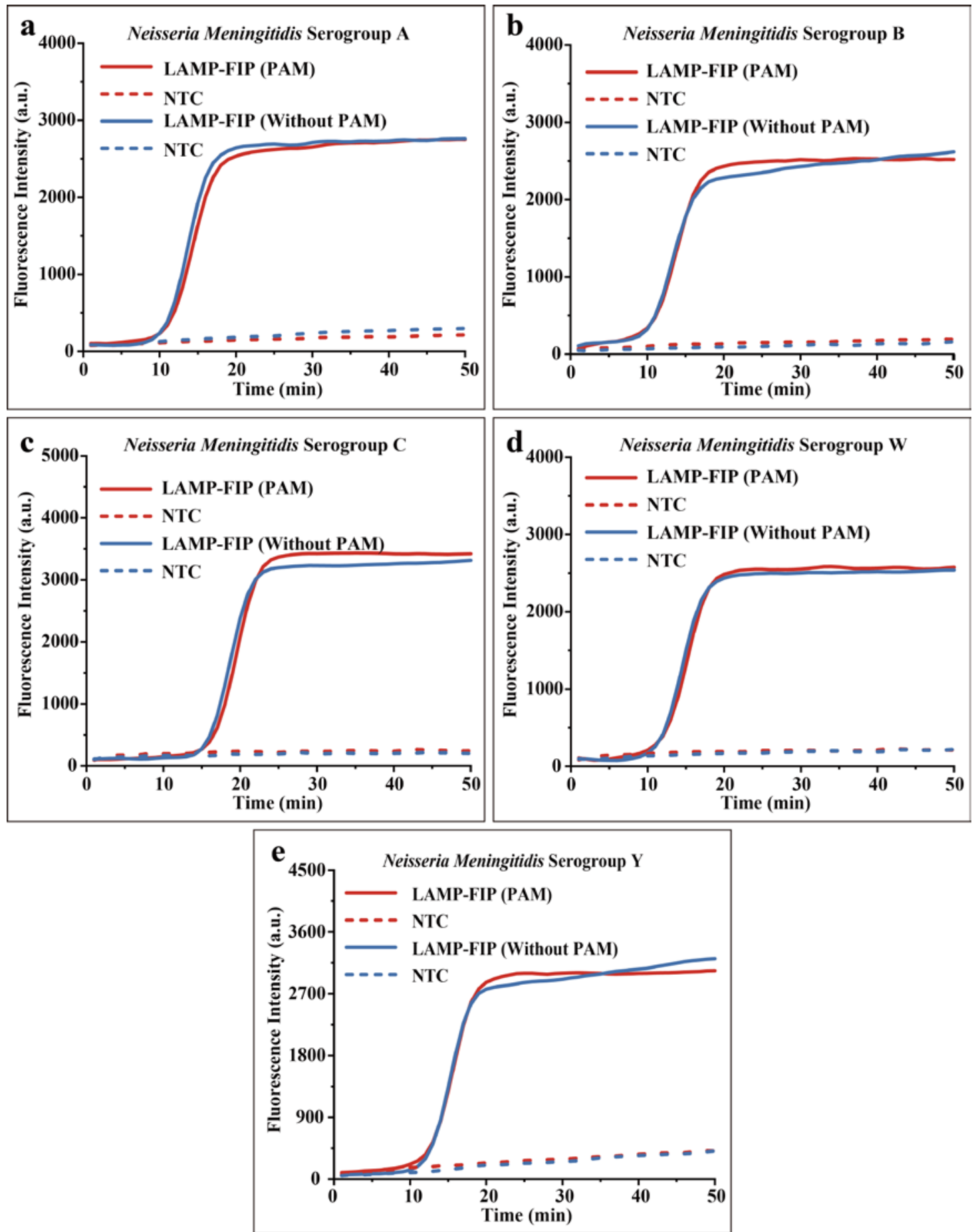

Figure S6. Test of the CON-LAMP amplification efficiency and specificity by using conventional FIPs or BIPs without PAM site and modified FIPs or BIPs with PAM site. (a) to (e) represent the LAMP amplification of ctrA gene for all five kinds of $N$. meningitidis serogroups (A, B, C, W, and Y) by using conventional primer without PAM site and modified primer with PAM site. NTC stands for no template control. 

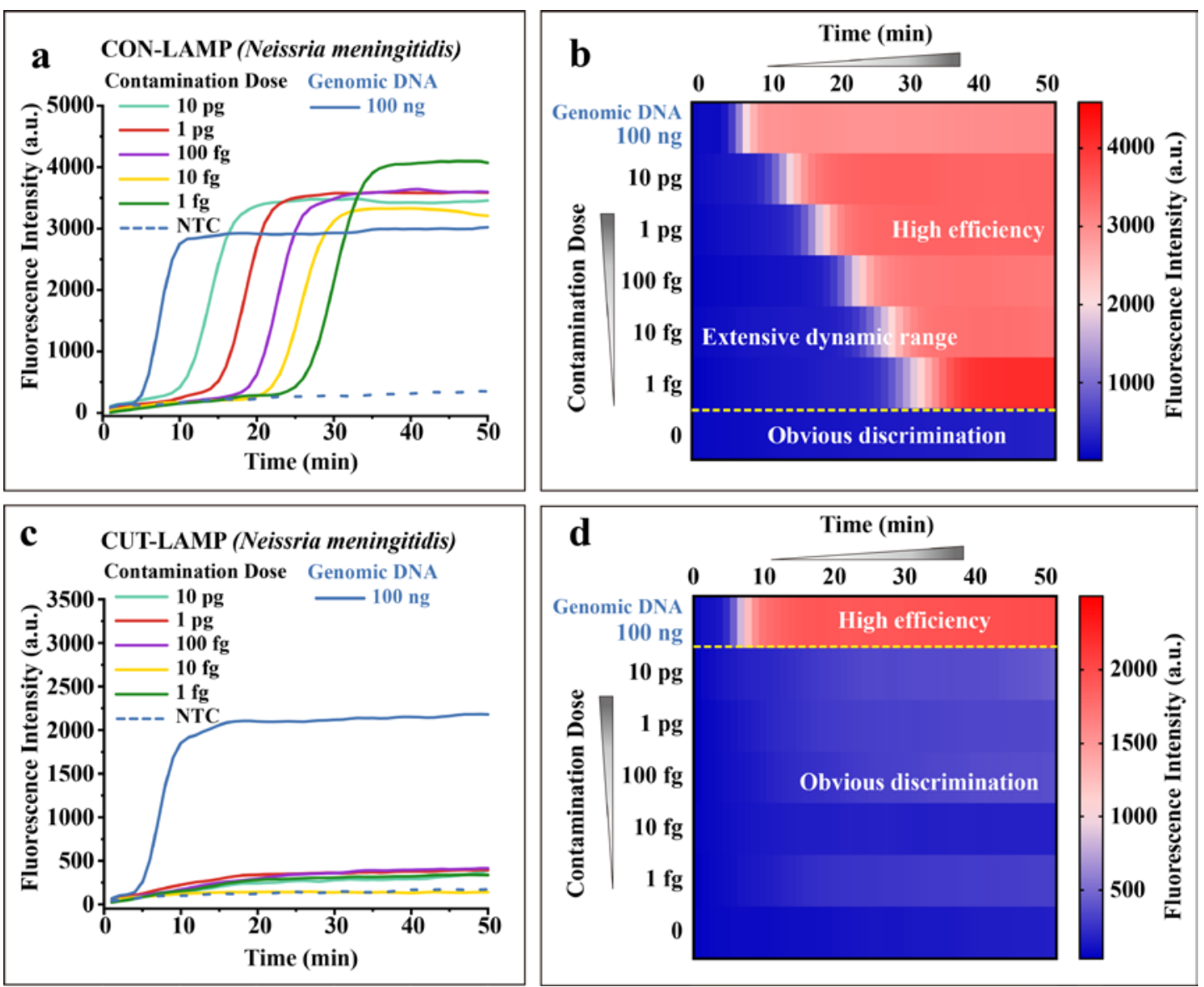

Figure S7. Contamination resistance of the LAMP reactions. (a) Contamination resistance of the CON-LAMP reaction. Genomic DNA as a positive control. (b) The dynamic range of the quantification of contamination and genomic DNA. (c) Contamination resistance of the CONLAMP reaction. Genomic DNA as a positive control. (d) The dynamic range of the quantification of contamination and genomic DNA. All experiments are based on the amplification of the ctrA gene from $N$. meningitidis. NTC stands for no template control. 


\section{Supporting Tables}

Table S1. Oligonucleotides used for the detection of invA gene of Salmonella. ${ }^{a}$

\begin{tabular}{|l|l|}
\hline \multicolumn{1}{|c|}{ Nucleic Acids ID } & \multicolumn{1}{c|}{ Sequences (5’-3’) } \\
\hline Primer set for CON-LAMP (invA gene of Salmonella) \\
\hline Sal-FIP & GACGACTGGTACTGATCGATAGTTTTTCAACGTTTCCTGCGG \\
\hline Sal-BIP & CCGGTGAAATTATCGCCACACAAAACCCACCGCCAGG \\
\hline Sal-FOP & GGCGATATTGGTGTTTATGGGG \\
\hline Sal-BOP & AACGATAAACTGGACCACGG \\
\hline Sal-LF & GACGAAAGAGCGTGGTAATTAAC \\
\hline Sal-LB & GGGCAATTCGTTATTGGCGATAG \\
\hline $\begin{array}{l}\text { Primer set for CUT-LAMP (invA gene of Salmonella). } \\
\text { Sal-BIP, Sal-FOP, Sal-FOP, Sal-BOP, Sal-LF, and Sal-LB are the same as CON-LAMP }\end{array}$ \\
\hline Sal-FIP & GACGACTGGTACTGATCGATCCAGTTTTTCAACGTTTCCTGCGG \\
\hline Primer set for synthesizing sgRNA for CUT-LAMP (invA gene of Salmonella) \\
\hline \multirow{2}{|l|}{ sgRNA-F (Sal-FIP) } & $\begin{array}{l}\text { GAAATTAATACGACTCACTATAGGCGCAGGAAACGTTGAAAAACG } \\
\text { TTTTAGAGCTAGAAATAGC }\end{array}$ \\
\hline sgRNA-R & $\begin{array}{l}\text { AAAAGCACCGACTCGGTGCCACTTTTTCAAGTTGATAACGGACTAG } \\
\text { CCTTATTTTAACTTGCTATTTCTAGCTCTAAAAC }\end{array}$ \\
\hline
\end{tabular}

${ }^{a}$ The nucleotide for generating PAM site is highlighted in yellow. sgRNA-R represents a common sgRNA reverse primer for generating sgRNA backbone. 
Table S2. Oligonucleotides used for the CRIPSR/Cas9 cleavage of EGFP.

\begin{tabular}{|c|c|}
\hline Nucleic Acids ID & Sequences (5’-3') \\
\hline \multicolumn{2}{|c|}{ Primer set for amplifying EGFP gene } \\
\hline$E G F P-F$ & ATGGTGAGCAAGGGCGAG \\
\hline EGFP-R & TTACTTGTACAGCTCGTCCATGC \\
\hline \multicolumn{2}{|c|}{ Primer set for synthesizing sgRNA for targeting EGFP } \\
\hline sgRNA-F (EGFP) & $\begin{array}{l}\text { GAAATTAATACGACTCACTATAGGGAAGGAGGACGGCAACATCC } \\
\text { TGTTTTAGAGCTAGAAATAGC }\end{array}$ \\
\hline $\operatorname{sgRNA-R}(E G F P)$ & $\begin{array}{l}\text { AAAAGCACCGACTCGGTGCCACTTTTTCAAGTTGATAACGGACTA } \\
\text { GCCTTATTTTAACTTGCTATTTCTAGCTCTAAAAC }\end{array}$ \\
\hline
\end{tabular}

${ }^{b}$ sgRNA-R represents a common sgRNA reverse primer for generating sgRNA backbone. 
Table S3. Oligonucleotides used for the detection of serogroups $\mathrm{A}, \mathrm{B}, \mathrm{C}, \mathrm{W}$, and $\mathrm{Y}$ of $N$. meningitidis. $^{c}$

\begin{tabular}{|c|c|}
\hline Nucleic Acids ID & Sequences (5’-3’) \\
\hline \multicolumn{2}{|c|}{ Primer set for CON-LAMP (sacB gene of $N$. meningitidis serogroup A) } \\
\hline Nm-A-FIP & $\begin{array}{l}\text { GAACTCTAATCTGAACCAAAATTGAGAGTTGACATGAAACTCAGCAC } \\
\text { AG }\end{array}$ \\
\hline Nm-A-BIP & CCTACAGCTAACAGATATTCTAGAAAACGAATAGTTTCGTATGCCTTC \\
\hline Nm-A-FOP & CGTAAATGAAATTTGGACAG \\
\hline Nm-A-BOP & TTATGATCTTCTTCATAGGGTA \\
\hline Nm-A-LF & ATAGATGAACTTAAAGTTCT \\
\hline Nm-A-LB & GGAAGCACTCTATTAAAAATAATC \\
\hline \multicolumn{2}{|c|}{$\begin{array}{l}\text { Primer set for CUT-LAMP (sacB gene of } N \text {. meningitidis serogroup A) } \\
\text { Nm-A-BIP, Nm-A-FOP, Nm-A-BOP, Nm-A-LF, and Nm-A-LB are the same as CON-LAMP }\end{array}$} \\
\hline Nm-A-FIP (PAM) & $\begin{array}{l}\text { GAACTCTAATCTGAACCAAAATTGAGAGTTCCGACATGAAACTCAGC } \\
\text { ACAG }\end{array}$ \\
\hline \multicolumn{2}{|c|}{ Primer set for synthesizing sgRNA for CUT-LAMP (sacB gene of $N$. meningitidis serogroup A) } \\
\hline sgRNA-F (Nm-A-FIP) & $\begin{array}{l}\text { GAAATTAATACGACTCACTATAGGCTGTGCTGAGTTTCATGTGTTTTA } \\
\text { GAGCTAGAAATAGC }\end{array}$ \\
\hline sgRNA-R & $\begin{array}{l}\text { AAAAGCACCGACTCGGTGCCACTTTTTCAAGTTGATAACGGACTAGCC } \\
\text { TTATTTTAACTTGCTATTTCTAGCTCTAAAAC }\end{array}$ \\
\hline \multicolumn{2}{|c|}{ Primer set for CON-LAMP (siaD gene of $N$. meningitidis serogroup B) } \\
\hline Nm-B-FIP & GGCCAGGCCTATAATTCCTTCCTTTTCTAATTGAGCCCCT \\
\hline Nm-B-BIP & САСССТСААСССААТGTСТTTСТСАТTTCAGTGTTTTCСАСС \\
\hline Nm-B-FOP & AAACCCTCGGCTGGTAG \\
\hline Nm-B-BOP & CTTAATAATCTCTAAGTGTTCTTG \\
\hline Nm-B-LB & GGAGAGTTAATTATTAACTTAATTCAA \\
\hline \multicolumn{2}{|c|}{$\begin{array}{l}\text { Primer set for CUT-LAMP (siaD gene of } N \text {. meningitidis serogroup B) } \\
\text { Nm-B-FIP, Nm-B-FOP, Nm-B-BOP, and Nm-B-LB are the same as CON-LAMP }\end{array}$} \\
\hline Nm-B-BIP (PAM) & CACССТСAАСССАATGTCСТTTCTCATTTCAGTGTTTTCСACС \\
\hline \multicolumn{2}{|c|}{ Primer set for synthesizing sgRNA for CUT-LAMP (siaD gene of $N$. meningitidis serogroup B) } \\
\hline sgRNA-F (Nm-B-BIP) & $\begin{array}{l}\text { GAAATTAATACGACTCACTATAGGAAAACACTGAAATGAGAAGTTTT } \\
\text { AGAGCTAGAAATAGC }\end{array}$ \\
\hline sgRNA-R & $\begin{array}{l}\text { AAAAGCACCGACTCGGTGCCACTTTTTCAAGTTGATAACGGACTAGCC } \\
\text { TTATTTTAACTTGCTATTTCTAGCTCTAAAAC }\end{array}$ \\
\hline \multicolumn{2}{|c|}{ Primer set for CON-LAMP (siaD gene of N. meningitidis serogroup C) } \\
\hline Nm-C-FIP & CCTACTACCCAATGTCTGTCAATTTTGTTGGGCTGTATGGTG \\
\hline Nm-C-BIP & AGTCGATGTCAGTCCAATAATTCCTGTAGTGATTAATGAACCCCCT \\
\hline Nm-C-FOP & TGCTCTTCAATTAAAGCGG \\
\hline $\mathrm{Nm}-\mathrm{C}-\mathrm{BOP}$ & GGTAACAATTAATCCCCGTCT \\
\hline Nm-C-LF & GGGCAAATCGTGATTG \\
\hline Nm-C-LB & GGGTTGTTAAATAAATTAGTGG \\
\hline \multicolumn{2}{|c|}{$\begin{array}{l}\text { Primer set for CUT-LAMP (siaD gene of } N \text {. meningitidis serogroup C) } \\
\text { Nm-C-BIP, Nm-C-FOP, Nm-C-BOP, Nm-C-LF, and Nm-C-LB are the same as CON-LAMP }\end{array}$} \\
\hline Nm-C-FIP (PAM) & CСTACTACCCAATGTCTGTCAATTCCATTGTTGGGCTGTATGGTG \\
\hline \multicolumn{2}{|c|}{ Primer set for synthesizing sgRNA for CUT-LAMP (siaD gene of $N$. meningitidis serogroup C) } \\
\hline sgRNA-F (Nm-C-FIP) & $\begin{array}{l}\text { GAAATTAATACGACTCACTATAGGCACCATACAGCCCAACAAGTTTTA } \\
\text { GAGCTAGAAATAGC }\end{array}$ \\
\hline sgRNA-R & $\begin{array}{l}\text { AAAAGCACCGACTCGGTGCCACTTTTTCAAGTTGATAACGGACTAGCC } \\
\text { TTATTTTAACTTGCTATTTCTAGCTCTAAAAC }\end{array}$ \\
\hline \multicolumn{2}{|c|}{ Primer set for CON-LAMP (synG gene of $N$. meningitidis serogroup W) } \\
\hline Nm-W-FIP & CTCACTTTCTGATGTCATGATCAGGTTATTCAAAGGTGAATCTTCCGA \\
\hline
\end{tabular}




\begin{tabular}{|c|c|}
\hline Nm-W-BIP & GGAAGGCATGGTGTATGATATTCCGTTACTGTAATCATTCGCTCC \\
\hline Nm-W-FOP & GACAATAAGTTACAAAACCGTATC \\
\hline Nm-W-BOP & TCACCAGTTTTAAAAACACAACC \\
\hline Nm-W-LB & TCTGTATTTTCATAAATTTCCTGC \\
\hline \multicolumn{2}{|c|}{$\begin{array}{l}\text { Primer set for CUT-LAMP (synG gene of } N \text {. meningitidis serogroup } \mathrm{W} \text { ) } \\
\text { Nm-W-BIP, Nm-W-FOP, Nm-W-BOP, and Nm-W-LB are the same as CON-LAMP }\end{array}$} \\
\hline Nm-W-FIP (PAM) & $\begin{array}{l}\text { CTCACTTTCTGATGTCATGATCAGGCCTTATTCAAAGGTGAATCTTCCG } \\
\text { A }\end{array}$ \\
\hline \multicolumn{2}{|c|}{ Primer set for synthesizing sgRNA for CUT-LAMP (synG gene of $N$. meningitidis serogroup W) } \\
\hline sgRNA-F (Nm-W-FIP) & $\begin{array}{l}\text { GAAATTAATACGACTCACTATAGGAAGATTCACCTTTGAATAGTTTTA } \\
\text { GAGCTAGAAATAGC }\end{array}$ \\
\hline sgRNA-R & $\begin{array}{l}\text { AAAAGCACCGACTCGGTGCCACTTTTTCAAGTTGATAACGGACTAGCC } \\
\text { TTATTTTAACTTGCTATTTCTAGCTCTAAAAC }\end{array}$ \\
\hline \multicolumn{2}{|c|}{ Primer set for CON-LAMP (synF gene of $N$. meningitidis serogroup Y) } \\
\hline Nm-Y-BIP & CACTGCCCACTATAAGCATGTTTTGAGTTGAAGAGGATGAGTGA \\
\hline Nm-Y-FIP & CGGGTTTGAAGAATTGTTGATGGTGACATTCCAGAAAATGTTAG \\
\hline Nm-Y-FOP & TGTCAAAACCTCCAGC \\
\hline Nm-Y-BOP & CGCTAAACGATACATTTCCA \\
\hline Nm-Y-LF & GAATAAAAAGGAATATTTCGGC \\
\hline Nm-Y-LB & TCTTTATTATCTGAAGAAGATAGC \\
\hline \multicolumn{2}{|c|}{$\begin{array}{l}\text { Primer set for CUT-LAMP (synF gene of } N \text {. meningitidis serogroup Y) } \\
\text { Nm-Y-FIP, Nm-Y-FOP, Nm-Y-BOP, Nm-Y-LF, and Nm-Y-LB are the same as CON-LAMP }\end{array}$} \\
\hline Nm-Y-BIP (PAM) & CACTGCCCACTATAAGCATGTTTTGCCAGTTGAAGAGGATGAGTGA \\
\hline \multicolumn{2}{|c|}{ Primer set for synthesizing sgRNA for CUT-LAMP (synF gene of $N$. meningitidis serogroup Y) } \\
\hline sgRNA-F (Nm-Y-BIP) & $\begin{array}{l}\text { GAAATTAATACGACTCACTATAGGTCACTCATCCTCTTCAACGTTTTA } \\
\text { GAGCTAGAAATAGC }\end{array}$ \\
\hline sgRNA-R & $\begin{array}{l}\text { AAAAGCACCGACTCGGTGCCACTTTTTCAAGTTGATAACGGACTAGCC } \\
\text { TTATTTTAACTTGCTATTTCTAGCTCTAAAAC }\end{array}$ \\
\hline
\end{tabular}

${ }^{c}$ The nucleotide for generating PAM sites are highlighted in yellow. sgRNA-R represents a common sgRNA reverse primer for generating sgRNA backbone. 
Table S4. Oligonucleotides used for the detection of $\operatorname{ctr} A$ gene of $N$. meningitidis. ${ }^{d}$

\begin{tabular}{|l|l|}
\hline \multicolumn{1}{|c|}{ Nucleic Acids ID } & \multicolumn{1}{c|}{ Sequences (5'-3') } \\
\hline Primer set for CON-LAMP (ctrA gene of $\boldsymbol{N}$. meningitidis) \\
\hline Nm-ctrA-FIP & CAAACACACCACGCGCATCAGATCTGAAGCCATTGGCCGTA \\
\hline Nm-ctrA-BIP & TGTTCCGCTATACGCCATTGGTACTGCCATAACCTTGAGCAA \\
\hline Nm-ctrA-FOP & AGCYAGAGGCTTATCGCTT \\
\hline Nm-ctrA-BOP & ATACCGTTGGAATCTCTGCC \\
\hline Nm-ctrA-LF & CGATCTTGCAAACCGCCC \\
\hline Nm-ctrA-LB & GCAGAACGTCAGGATAAATGGA \\
\hline $\begin{array}{l}\text { Primer set for CUT-LAMP (ctrA gene of } \text { N. } \text { meningitidis) } \\
\text { Nm-ctrA-BIP, Nm-ctrA-FOP, Nm-ctrA-FOP, Nm-ctrA-BOP, Nm-ctrA-LF, and Nm-ctrA-LB are the } \\
\text { same as CON-LAMP }\end{array}$ \\
\hline Nm-ctrA-FIP (PAM) & CAAACACACCACGCGCATCAGACCTCTGAAGCCATTGGCCGTA \\
\hline Primer set for synthesizing sgRNA for CUT-LAMP (ctrA gene of N. meningitidis) \\
\hline $\begin{array}{l}\text { sgRNA-F-ctrA-FIP } \\
\text { (PAM) }\end{array}$ & $\begin{array}{l}\text { GAAATTAATACGACTCACTATAGGGTACGGCCAATGGCTTCAGGTTTT } \\
\text { AGAGCTAGAAATAGC }\end{array}$ \\
\hline sgRNA-R & $\begin{array}{l}\text { AAAAGCACCGACTCGGTGCCACTTTTTCAAGTTGATAACGGACTAGCC } \\
\text { TTATTTTAACTTGCTATTTCTAGCTCTAAAAC }\end{array}$ \\
\hline
\end{tabular}

${ }^{d}$ The nucleotide for generating PAM site is highlighted in yellow. sgRNA-R represents a common sgRNA reverse primer for generating sgRNA backbone. 
Table S5. Oligonucleotides used for the detection of Zika virus. ${ }^{e}$

\begin{tabular}{|c|c|}
\hline Nucleic Acids ID & Sequences (5'-3') \\
\hline \multicolumn{2}{|c|}{ Primer set for CON-LAMP (Zika virus) } \\
\hline Zika-MR766-FIP & GGCGGCATTTCAAATGGCCAGCTCGCTGGAGCTCTAGAGG \\
\hline Zika-MR766-BIP & TATTCCTTGTGCACTGCGGCATGACTGTTCCATGCAGTGTT \\
\hline Zika-MR766-FOP & GAAGGAGCCGTTCACACG \\
\hline Zika-MR766-BOP & CCTGCATACTGCACCTCC \\
\hline Zika-MR766-LF & CCTTTGCACCATCCATCTCAG \\
\hline Zika-MR766-LB & TTCACATTCACCAAGGTCCCA \\
\hline \multicolumn{2}{|c|}{$\begin{array}{l}\text { Primer set for CUT-LAMP (Zika virus) } \\
\text { Zika-MR766-FIP, Zika-MR766-FOP, Zika-MR766-BOP, Zika-MR766-LF, and Zika-MR766-LB are the } \\
\text { same as CON-LAMP }\end{array}$} \\
\hline Zika-BIP-PAM & TATTCCTTGTGCACTGCGGCACCTGACTGTTCCATGCAGTGTT \\
\hline \multicolumn{2}{|c|}{ Primer set for synthesizing sgRNA for CUT-LAMP (Zika virus) } \\
\hline sgRNA-F (Zika BIP) & $\begin{array}{l}\text { GAAATTAATACGACTCACTATAGGGAACACTGCATGGAACAGTCG } \\
\text { TTTTAGAGCTAGAAATAGC }\end{array}$ \\
\hline sgRNA-R & $\begin{array}{l}\text { AAAAGCACCGACTCGGTGCCACTTTTTCAAGTTGATAACGGACTA } \\
\text { GCCTTATTTTAACTTGCTATTTCTAGCTCTAAAAC }\end{array}$ \\
\hline
\end{tabular}

${ }^{e}$ The nucleotide for generating PAM site is highlighted in yellow. sgRNA-R represents a common sgRNA reverse primer for generating sgRNA backbone. 
Table S6. Oligonucleotides used for the CRISPR/Cas12a detection system.

\begin{tabular}{|c|c|}
\hline Nucleic Acids ID & Sequences (5’-3’) \\
\hline \multicolumn{2}{|c|}{ Template for synthesizing crRNA for targeting invA gene of Salmonella } \\
\hline Salmonella-invA-crRNA-1 & $\begin{array}{l}\text { GCCCTTAATACGACTCACTATAGGGAATTTCTACTGTTGTAGATCTG } \\
\text { CGGTACTGTTAATTACCACGC }\end{array}$ \\
\hline Salmonella-invA-crRNA-2 & $\begin{array}{l}\text { GCGTGGTAATTAACAGTACCGCAGATCTACAACAGTAGAAATTCCCT } \\
\text { ATAGTGAGTCGTATTAAGGGC }\end{array}$ \\
\hline \multicolumn{2}{|c|}{ Fluorescent probe used in CRISPR/Cas12a system } \\
\hline Probe & FAM-TTTGCTGCTGAGGAA-BHQ \\
\hline \multicolumn{2}{|c|}{ Primer set for CON-LAMP (ermC gene of MRSA) } \\
\hline ermC FIP & CCAGACGTTTGTTTACGGTATAACTCTCATAGACGAAGAAAGTGGAG \\
\hline ermC BIP & TGGCGGAAAAAAACTTAAAATCGTTGTATTGTTACTTAAGTGGACAT \\
\hline ermC FOP & GTCTATAAAGGCTCTCAGAGG \\
\hline ermC BOP & GCTATTTCTCTTGTTGTAGCTATC \\
\hline \multicolumn{2}{|l|}{ Gene sequence of ermC } \\
\hline $\begin{array}{l}\text { ermC }(2069 \sim 2365), \\
\text { GenBank accession NO. } \\
\text { V01278 }\end{array}$ & $\begin{array}{l}\text { AATCGTCAATTCCTGCATGTTTTAAGGAATTGTTAAATTGATTTTTTG } \\
\text { TAAATATTTTCTTGTATTCTTTGTTAACCCATTTCATAACGAAATAAT } \\
\text { TATACTTTTGTTTATCTTTGTGTGATATTCTTGATTTTTTTCTACTTAA } \\
\text { TCTGATAAGTGAGCTATTCACTTTAGGTTTAGGATGAAAATATTCTC } \\
\text { TTGGAACCATACTTAATATAGAAATATCAACTTCTGCCATTAAAAGT } \\
\text { AATGCCAATGAGCGTTTTGTATTTAATAATCTTTTAGCAAACCCGTA } \\
\text { TTCCACGATTA }\end{array}$ \\
\hline
\end{tabular}

